\section{AUDIT OF REFERRALS FROM OCCUPATIONAL HEALTH SERVICE TO PUBLIC HEALTH ENGLAND NATIONAL SURVEILLANCE SCHEME FOR OCCUPATIONAL EXPOSURE TO SIGNIFICANT BBV}

E McCarthy, E Murphy*. Newcastl OHS, The Newcastle upon Tyne NHS Hospitals Foundation Trust, Newcastle Upon Tyne, UK

\subsection{6/oemed-2018-ICOHabstracts.965}

Introduction Newcastle OHS submits data to the voluntary Public Health England (PHE) significant exposures surveillance system (SigOcc). Data from the Eye of the Needle report (2004 - 2013) showed that there were 4830 significant exposures to a blood borne virus (BBV) reported in healthcare workers and that there was an increase in reported numbers over this time period. ${ }^{1}$

The audit aim was to review the quality of data contained in forms returned to NewcastleOHS from PHE between January 2010 and December 2015 following initial submissions.

Methods Cycle 1 - A retrospective audit was carried out to analyse the data collected from PHE forms returned between January 2010 and December 2015. Significant exposures were documented on PHE paper forms which were analysed against the above standards

Cycle 2 - A prospective audit of data between December 2015 and December 2016 was carried out. Each case was allocated to a clinician who was responsible for reviewing results and completing the forms at 0,6 and 24 weeks. This data was collected and entered electronically on forms for submission by email

Result Cycle 1-26 employees had been reported to SigOcc as having had a significant exposure to a BBV detected source. Follow up was completed in $80 \%$

Cycle 2-21 employees had been reported to OHS as having has a significant exposure to a BBV detected source (December 2015 - 16). On review of these exposures, 86\% (18) were reported to SigOcc as data indicated a very low risk exposure in 3 cases. Follow up was 100\% in cycle 2

Discussion HIV exposure in cycle 1 of the audit was higher than reported in the Eye of the Needle report at 54\%. In cycle 2 the rate was 33\%, similar to published studies. There were 14 exposures to HIV reported to SigOcc in the North East in 2014-15. For HIV infection standard reporting levels were based on HIV antibody detection. HIV PCR levels were not always reported $(54 \%$ had documented viral loads or CD4 counts). Sig Occ recommends referral of all exposures to HIV antibody detected body fluids. This may need review given new treatments and viral suppression.

Hepatitis C exposure in cycle 1 of the audit was 34\%, increasing to $48 \%$ in cycle 2 , similar to reported rates. There were 18 reported exposures to Hepatitis $\mathrm{C}$ to SigOcc in the North East in 2014- 5 (51\%). Hepatitis C PCR was documented in $67 \%$ of cases in cycle 1 . SigOcc recommend referral of all hepatitis $\mathrm{C}$ antibody detected exposure, however this may require review given viral suppression treatment.

Hepatitis B exposures were $8 \%$ in cycle $1 \%$ and $19 \%$ in cycle 2. There were 5 reported cases to SigOcc in the North East in 2014 - 15. Hepatitis B testing appears to be more complete with hepatitis B surface antigen levels and viral DNA level available in known exposure cases.

\section{6 EXPOSURE TO ALDEHYDES AMONG HEALTH CARE WORKERS IN A LARGE TERTIARY HOSPITAL IN CAPE TOWN, SOUTH AFRICA}

${ }^{1,2} \mathrm{HH}$ Mwanga*, ${ }^{1,3} \mathrm{R}$ Baatjies, ${ }^{1} \mathrm{MF}$ Jeebhay. ${ }^{1}$ Centre for Environmental and Occupational Health Research and Division of Occupational Medicine, School of Public Health and Family Medicine, University of Cape Town, Cape Town, South Africa; 'Department of Environmental and Occupational Health, School of Public Health and Social Sciences, Muhimbili University of Health and Allied Sciences, Dar es Salaam, Tanzania; ${ }^{3}$ Department of Environmental and Occupational Studies, Faculty of Applied Sciences, Cape Peninsula University of Technology, Cape Town, South Africa

\subsection{6/oemed-2018-ICOHabstracts.966}

Introduction High-level disinfectants such as ortho-phthalaldehyde (OPA) have increasingly been linked to various adverse health outcomes including occupational asthma, contact dermatitis and anaphylaxis. However, environmental exposure characterisation studies of aldehydes such as OPA, are limited. The aim of this study was to conduct a detailed exposure characterisation study of health care workers (HCWs) exposed to aldehydes.

Methods A total of 269 full-shift passive personal samples were collected from HCWs randomly selected from 17 different clinical departments in a tertiary hospital. Passive sampling used TraceAir AT580 monitors (Assay Technology, Livermore, CA), which were analysed for OPA and formaldehyde.

Result OPA was detectable in $6(2 \%)$ samples from gastrointestinal (GI) unit, with a median of 0.009 ppm (range: $0.005-$ 0.027). HCWs with detectable OPA levels had a longer duration of OPA use $(\mathrm{OR}=1.28 ; 95 \% \mathrm{CI}: 1.10$ to 1.50$)$. Formaldehyde was detectable in $103(38 \%)$ samples with a median of $0.004 \mathrm{ppm}$ (range: 0.003-0.027). Three (1\%) samples had formaldehyde levels higher than the NIOSH recommended exposure limit (REL) of $0.016 \mathrm{ppm}$ time-weighted average.

Discussion In this study, OPA levels were on average 10-fold higher than in similar settings elsewhere. Detectable OPA in the GI unit corroborates findings of workplace inspections conducted during the exposure measurements, which found high level of OPA usage among sterilising operators and registered nurses. Formaldehyde levels were on average 10-fold lower than studies in pathology and anatomical laboratories elsewhere but were comparable to average levels in US general buildings. The most likely source of exposure is probably related to exposure to formaldehyde (10\%) solution used for specimen preparation in most departments, residue evaporation from formaldehyde contaminated surfaces and other general indoor sources. The study concluded that mean detectable exposures to OPA are higher and more isolated than more widespread low-level formaldehyde exposures.

\section{OCCUPATIONAL HEALTH AND SAFETY MANAGEMENT IN THAI HOSPITAL}

${ }^{1}$ Noppanun Nankongnab*, ${ }^{1}$ Pornpimol Kongtip, ${ }^{2}$ Susan Woskie. ${ }^{1}$ Department of Occupational Health and Safety, Faculty of Public Health, Mahidol University, Bangkok, Thailand; ${ }^{2}$ College of Health Sciences, University of Massachusetts Lowell, Lowell, MA, USA

\subsection{6/oemed-2018-ICOHabstracts.967}

Introduction Statistic showed that hospital is the most hazardous workplace. More than 300000 healthcare workers were 
employed in approximately 2627 public and 321 private hospitals in Thailand.

Methods The cross-sectional study aimed to investigate the occupational health and safety management in five Thai hospitals. The questionnaire consisted of characteristic of healthcare workers both working directly with patient such as inpatient, out- patient and not working directly with patients such as nutrition service and hospital supporting service departments, accident statistics and occupational health and safety management.

Results The result showed that healthcare workers in inpatient department had highest percentage reporting all 29 items of occupational health and safety management such as having safety policy, communication of policy to staff, having safety committee meeting, alarm system preparedness, fire prevention and protection preparedness, personal protective equipment provided, etc. followed by out-patient, nutrition service and hospital supporting service departments. The accident in the past three months indicated that the accident of sharp injury and falling from slippery floor in the nutrition service were $57.7 \%$ and $26.9 \%$, respectively. Furthermore, the comparison of the accident of sharp injury and falling from slippery floor of nutrition service with other departments was statistically significant different $(\mathrm{p}<0.001)$. The accident of needle stick and contact with chemicals, body fluid in surgery and anaesthesia department were $26.9 \%$ and $11.5 \%$, respectively. The comparison of needle stick and contact with chemicals, body fluid of surgery and anaesthesia department with other departments was statistically significant different that were $\mathrm{p}<0.001$ and $\mathrm{p}=0.024$, respectively.

Conclusion The situations of occupational health and safety management in Thai hospital were crucial for problem recognition and making the further control measure

\section{OCCUPATIONAL STRESS AND DEPRESSIVE SYMPTOMS IN HOSPITAL DOCTORS WORKING IN IRELAND}

${ }^{1}$ Anthony O' Keeffe*, Blánaid Hayes ${ }^{1},{ }^{2}$ Lucia Prihodova. ${ }^{1}$ Faculty of Occupational Medicine, $R C P I$, Dublin, Ireland; ${ }^{2}$ Research department, RCPI, Dublin, Ireland

\subsection{6/oemed-2018-ICOHabstracts.968}

Background Depression is reported to be prevalent amongst doctors and can have serious implications on the individual and on patient care. Previous studies have demonstrated an association between occupational stress (OS) and depressive symptoms amongst doctors. This study assessed the association between OS and depressive symptoms in hospital doctors in Ireland.

Methods In this randomised cross-sectional survey, 1749 doctors (950 consultants, 799 trainees) provided data on demographics (age, sex) and work (specialty, grade, working hours). Effort Reward Imbalance questionnaire was used to assess OS (effort-reward ratio [ERR], overcommitment [OC]). The WHO-5 Wellbeing index was used to assess depressive symptoms (two categories: 'normal/mild' vs 'likely depression'). Chi-square tests and t-tests were used to test differences in depressive symptoms and OS for sex, work grade and specialty. Binary logistic regression was performed to determine the association between OS and depressive symptoms, while controlling for sex, age, grade, specialty and mean hours worked.
Results Compared to males, females reported higher mean ERR and OC. Trainees reported higher mean ERR, OC and higher prevalence of 'likely depression' compared with consultants $(27.4 \%-\mathrm{v}-17.8 \%, \mathrm{p}=0.000)$. Those in the upper ERR tertile were more likely to experience depressive symptoms than those in the lowe tertile $(\mathrm{OR}=3.723$; CI: 2.634 to $5.263 ; \mathrm{p}=0.000)$. Those in the upper OC tertile were significantly more likely to experience depressive symptoms than those in the lowest tertile $(\mathrm{OR}=4.003$; CI: 2.87 to 5.884; $\mathrm{p}=0.000)$. Mean hours worked $(\mathrm{OR}=1.02 ; \mathrm{CI} ; 1.009$ to 1.03 ; $\mathrm{p}=0.000$ ) and grade (consultants $[\mathrm{OR}=0.496 ; \mathrm{CI}: 0.292$ to $0.843 ; \mathrm{p}=0.01]$ ) were significantly associated with depressive symptoms, whereas age, sex and specialty were not. The model explained $18 \%$ of variance.

Discussion OS and work conditions are associated with depressive symptoms in hospital doctors in Ireland. Efforts to reduce OS, such as review of work conditions or occupational social support, should be undertaken to improve psychological wellbeing.

\section{A SAMPLE TO DETERMINE THE CONDITIONS AND PROBLEMS FACED BY DISABLED HEALTH CARE PROFESSIONALS: A TRAINING AND RESEARCH HOSPITAL}

M Picakciefe, V Akkaya, E Erbas. Department of Public Health, Faculty of Medicine, Mugla Sitki Kocman University, Mugla, Turkey

\subsection{6/oemed-2018-ICOHabstracts.969}

Introduction It is predicted that worldwide, there are over one billion people who live with one sort of disability or another. The objective of this study was to determine the conditions and the problems faced by disabled health care professionals working in a training and research hospital.

Methods The method is a cross-sectional and definitive study. The population of study was comprised of 908 health care workers in a training and research hospital in Mugla, Turkey. A list was obtained from the human resources department of the hospital management and 323 people (accessibility rate $35.5 \%$ ) agreed to participate in a survey to determine disability. A brief set of questions prepared by the Washington Disability Statistics Group was implemented. The set results defined 63 people as being disabled (19.5\%).

Result The findings indicated that among the disabled health care workers, $44.5 \%$ were older (above the age of 40 ), $65.5 \%$ were females, $60.3 \%$ had a educational status of university or less, $38.1 \%$ were doctors, and $52.4 \%$ worked in the clinical sciences. $13.0 \%$ of the health care workers had cognitive impairment. $15.9 \%$ suffered from cardiac or circulatory ailments, $85.7 \%$ had complained of fatigue, and $73.0 \%$ had muscular-joint pains. In addition, $77.8 \%$ of the health care workers indicated that they had difficulties in commutes to and from the hospital in terms of the routes and the means of transport available. $65.1 \%$ claimed they found the breaks to be insufficient, $58.7 \%$ said they were victims of mobbing, $57.1 \%$ stated they desired to work part-time, and $55.6 \%$ indicated they worked in positions which did not necessitate any skill sets.

Discussion It appears disabled health care professionals face a number of negativities. A common set of definitions and a disability detection form should be established and prepared in 